\title{
Specific expression of proton-coupled oligopeptide transporter 1 in primary hepatocarcinoma-a novel strategy for tumor-targeted therapy
}

\author{
YANXIA GONG $^{1,2}$, JIE ZHANG $^{1}$, XIANG WU $^{3}$, TAO WANG $^{1}$, JIA ZHAO $^{4}$, \\ ZHI YAO ${ }^{5}$, QINGYU ZHANG ${ }^{1}, \mathrm{XI} \mathrm{LIU}^{1}$ and XU JIAN ${ }^{3}$ \\ ${ }^{1}$ Department of Gastroenterology, Tianjin Medical University General Hospital, Tianjin 300052; \\ ${ }^{2}$ Department of Gastroenterology, Tianjin Nankai Hospital, Tianjin 300100; ${ }^{3}$ Central Laboratory and ${ }^{4}$ Clinical Laboratory, \\ Tianjin Medical University General Hospital, Tianjin 300052; \\ ${ }^{5}$ Department of Immunology, School of Basic Medical Science, Tianjin Medical University, Tianjin 300070, P.R. China
}

Received December 11, 2015; Accepted May 16, 2017

DOI: $10.3892 / 01.2017 .6724$

\begin{abstract}
Proton-coupled oligopeptide transporter 1 (PEPT1) is a membrane protein which expressed predominantly in intestine and recognized as the target of dietary nutrients (di/tripeptide) or peptidomimetic drug for delivery. The information on the existence of PEPT1 in carcinomas were limited. Our study aimed to investigate the expression profile and transport activity of PEPT1 both in human hepatocarcinoma tissues and cell lines. Western blotting and an immunofluorescence assay revealed the high level of PEPT1 protein expression in hepatocarcinoma Bel-7402, SMMC-7721, HepG2, HEP3B, SK-HEP-1 cell lines. Quantitative real time PCR showed the mRNA expression of PEPT1 in Bel-7402, SMMC-7721, HepG2, HEP3B, SK-HEP-1 cells. High level PEPT1 expression in hepatocarcinoma patient samples were observed by Immunohistology and showed a significant correlation between protein level and pathological grade. Functional activities were also studied using D-Ala-Lys-AMCA (a substrate of peptide transporter) in above five hepatocarcinoma cell lines. The uptake tests performed by fluorescent microscopy suggested that PEPT1 can transport both D-Ala-Lys-AMCA into the hepatocarcinoma cells and the uptake can be competitively inhibited by three PEPT1 substrates (Gly-sar, Gly-gln and Glyglygly). In conclusion, our findings provided the novel information on the expression and function of PEPT1 in human hepatocarcinoma and expanded the potential values for tumor specific drug delivery.
\end{abstract}

Correspondence to: Dr Xu Jian, Central Laboratory, Tianjin Medical University General Hospital, 154 Anshan Road, Heping, Tianjin 300052, P.R. China

E-mail: jianxu516@hotmail.com

Key words: proton-coupled oligopeptide transporter 1, primary hepatocarcinoma, expression, tumor-targeted therapy

\section{Introduction}

Primary hepatocarcinoma, which arises from liver cells or intrahepatic bile duct epithelial cells, is one of the most fatal types of malignant tumors worldwide (1), causing 250,000 to $1,000,000$ mortalities per year, and has become the fifth and seventh most common malignant tumor in males and females, respectively (2). The majority of hepatocarcinoma cases occur in a number of developing countries (3). China has the highest morbidity and mortality associated with hepatocarcinoma; the number of patients with hepatocarcinoma in China accounts for $54 \%$ of the cases worldwide (4). The preferred treatment for hepatocarcinoma is liver resection, but this is restricted to early stages of hepatocarcinoma (5). Due to the difficulty of early detection, the rapid progression of the disease and the fact that the majority of patients exhibit liver cirrhosis, only a limited number of patients are able to undergo surgery, resulting in the majority of patients exhibiting a poor prognosis (6). Therefore, the identification of a specific tumor marker, for early diagnosis and targeted therapy, is required.

Proton-coupled oligopeptide transporter 1 (PEPT1) is one of the four members of the peptide transporter superfamily in mammalian cells (7). PEPT1 is expressed predominantly in the intestine and mediates the absorption of dietary nutrients (di/tripeptide) or peptidomimetic drugs (8). As PEPT1 is able to transport a broad spectrum of substrates, it is an attractive target for drug delivery (9). Previous studies on the presence and function of PEPT1 in carcinomas was limited in a number of carcinoma cell lines, including colon carcinoma Caco-2 cells (10), pancreatic carcinoma cell lines AsPc-1 and Capan-29 (11), gastric cancer (12), prostate cancer $(13,14)$ and fibroblast-derived tumor cells (15), which indicates that PEPT1 may be expressed in other types of cancer cells. The overexpression of PEPT1 in cancer cells may enable the identification of a specific pathway for therapeutic agents to exploit. Therefore, clarifying the expression patterns of PEPT1 in other types of cancer cell may expand the value of peptide transporters in cancer therapy. 
In the present study, the expression profile and function of PEPT1 in hepatocarcinoma were investigated in hepatocarcinoma cells and tissues. The results of the present study may provide novel information on the expression and function of PEPT1 in human hepatocarcinoma, and expand the values for hepatocarcinoma early diagnosis and tumor specific drug delivery.

\section{Materials and methods}

Materials. D-Ala-Lys-AMCA $(2.5 \mathrm{mg} / \mathrm{ml})$ was purchased from BIOTREND Chemikalien GmbH (Köln, Germany), and glycine (Gly)-sarcosine (Sar), Gly-glutamine (Gln) and Gly-Gly-Gly were obtained from Sigma-Aldrich(Merck KGaA, Darmstadt, Germany). The PCR primers used in the present study were synthesized by Integrated DNA Technologies, Inc. (Coralville, IA, USA). A rabbit polyclonal antibody against PEPT1 (anti-PEPT1 antibody; catalog no. 78020; dilution, 1:50) was provided by Abcam (Cambridge, UK). Additionally, the human liver cancer Bel-7402, SMMC7721, Hep3B, HepG2, and SK-HEP-1cell lines, the human gastric cancer BGC-823 cell line and the human colon cancer Caco-2 cell line were obtained from the Academia Sinica Cell Repository (Shanghai, China).

Cell culture. Bel-7402 cells were grown in RPMI-1640 medium (Gibco; Thermo Fisher Scientific, Inc., Waltham, MA, USA) containing $10 \%$ fetal bovine serum (FBS; Gibco; Thermo Fisher Scientific, Inc.), whereas SK-HEP-1 cells were cultured in Dulbecco's modified Eagle medium (DMEM; Gibco; Thermo Fisher Scientific, Inc.) medium containing 10\% FBS, $100 \mathrm{U} / \mathrm{ml}$ penicillin and $100 \mu \mathrm{g} / 1$ streptomycin. Concurrently, SMMC7721, Hep3B, HepG2 and Caco-2 cell lines were maintained in DMEM (Gibco; Thermo Fisher Scientific, Inc.) supplemented with 10\% FBS (Gibco; Thermo Fisher Scientific, Inc.), $1 \% 100 \mu \mathrm{g} / \mathrm{ml}$ penicillin (Sigma-Aldrich; Merck KGaA) and $100 \mu \mathrm{g} / \mathrm{ml}$ streptomycin (Sigma-Aldrich; Merck KGaA). All of the cells were maintained at $37^{\circ} \mathrm{C}$ in $5 \% \mathrm{CO}_{2}$. The culture media were changed every other day, and cells were passaged once they reached $80-90 \%$ confluence, at which point the cells were collected for protein or RNA extraction.

Immunofluorescence of cell lines. Subsequent to attachment onto a plate, $1 \times 10^{5}$ cells/ml (Bel-7402, SK-HEP-1, SMMC7721, Hep3B, HepG2 and Caco-2) were washed with medium, fixed with $4 \%$ paraformaldehyde for $5 \mathrm{~min}$ at room temperature, washed three times with PBS, treated with bovine serum albumin, (Wuhan Boster Biological Technology, Ltd., Wuhan, China) for $1 \mathrm{~h}$, and subsequently incubated with the rabbit anti-humananti-PEPT1 primary antibody (1:50 dilution) overnight at $4{ }^{\circ} \mathrm{C}$. Following three washes with PBS, the cells were incubated with a fluorescent goat anti-rabbit IgG secondary antibody (dilution, 1:100; catalog no. ZF036; Beijing ZSGB-BIO Co. Ltd., China) for $1 \mathrm{~h}$ at $37^{\circ} \mathrm{C}$, washed with PBS, stained with DAPI $(5 \mu \mathrm{g} / \mathrm{ml})$ for $5 \mathrm{~min}$ at room temperature, fixed, mounted, and observed using a fluorescent microscope (magnification, x20) to determine blue (nucleus) and red (PEPT1) fluorescence. Each experiment was repeated three times and Caco- 2 cell lines was used as the positive control.
Western blotting. All the cell types (Bel-7402, SK-HEP-1, SMMC7721, BGC-823, Hep3B, HepG2 and Caco-2) were seeded $\left(1 \times 10^{7}\right.$ cells $\left./ \mathrm{ml}\right)$ in $100-\mathrm{mm}$ cell culture dishes. Total protein was then extracted from the cells using $1 \%$ Nonidet P-40 lysis buffer (Sigma-Aldrich; Merck KGaA). The protein concentration was measured using the Lowry method. The protein lysates (30 $\mu \mathrm{g}$ protein/lane) were subsequently separated using $10 \%$ SDS-PAGE and transferred to polyvinylidene fluoride membranes (PVDF; EMD Millipore, Billerica, MA, USA). The blocking reagent used was $5 \%$ bicinchoninic acid, and the PVDF membranes were blocked at $37^{\circ} \mathrm{C}$ for $1 \mathrm{~h}$. These membranes were incubated with the primary antibody, as aforementioned (dilution, 1:100), followed by incubation with a horseradish peroxidase-conjugated secondary antibody (dilution, 1:100; catalog no. ZB-2301; Beijing ZSGB-BIO Co. Ltd) at room temperature. Protein expression was visualized using a Super Signal Protein Detection kit (Pierce; Thermo Fisher Scientific, Inc.). The membranes were then stripped and re-probed with anti-PEPT1 (dilution, 1:1,000), and an anti- $\beta$-actin primary antibody (dilution, 1:1,000; catalog no. sc-47778; Santa Cruz Biotechnology, Inc.) served as a loading control. Each experiment was repeated three times.

Reverse transcription-quantitative-polymerase chain reaction $(R T-q P C R)$ analysis. Total RNA was extracted from all of the cell types (Bel-7402, SK-HEP-1, SMMC7721, BGC-823, Hep3B, HepG2 and Caco-2) using the TRIzol reagent (Invitrogen; Thermo Fisher Scientific, Inc.) and then used for cDNA synthesis by RT with M-MLV Reverse Transcriptase (Promega Corporation, Madison, WI, USA) in a total volume of $10 \mu \mathrm{l}$ according to the manufacturer's protocol. The reverse transcription RT-PCR products were separated by electrophoresis on a $2 \%$ agarose gel. The gel was then stained with ethidium bromide, digitally photographed, and scanned with a UVI gel analysis system (UVItec, Cambridge, UK). Using a 7500 ABI RT-PCR machine (Applied Biosystems; Thermo Fisher Scientific, Inc.), expression levels were quantitatively analyzed. The TaqMan assay kit (Applied Biosystems; Thermo Fisher Scientific, Inc.) was used to detect gene expression. Quantification was also performed using amplification efficiencies derived from cDNA standard curves to obtain the relative gene expression. The data are presented as fold changes $\left(2^{-\Delta \Delta \mathrm{Cq}}\right)(16)$ and were initially analyzed using the Opticon Monitor V2.02 analysis software (MJ Research; Bio-Rad Laboratories, Inc., Hercules, CA, USA). Specific RT-PCR primers were obtained from Fulen Gene BioEngineering Inc. (Guangdong, China). The primers for PEPT1 were 5'-GCTCGGTTCTATACTTACATC-3' (forward) and 5'-TCCATCCTCCACTTGCCT-3' (reverse) (10). The primers for GAPDH as the reference gene were: 5'-AGGTCG GTGTGACGTTACGG-3' (forward) and 5'-GGGGTCGTT GATGGCAACAA-3' (reverse). Each was performed in triplicate. The thermocycling conditions were as follows: $95^{\circ} \mathrm{C}$ for $3 \mathrm{~min} ; 45 \mathrm{cycles}$ of $95^{\circ} \mathrm{C}$ for $15 \mathrm{sec}, 58^{\circ} \mathrm{C}$ for $30 \mathrm{sec}$ and $72^{\circ} \mathrm{C}$ for $30 \mathrm{sec}$; and $72^{\circ} \mathrm{C}$ for $2 \mathrm{~min}$.

Verification of D-Ala-Lys-AMCA. D-Ala-Lys-AMCA is a known PEPT1 substrate that emits blue fluorescence. All cell types were cultured in 24-well plates for 1 day, and the culture medium was then aspirated. Following one wash with PBS, D-Ala-Lys-AMCA (1 mM in PBS) was added for $2 \mathrm{~h}$ at $37^{\circ} \mathrm{C}$. 
D-Ala-Lys-AMCA fluorescence was then observed through a fluorescence microscope (magnification, x20).

The uptake of D-Ala-Lys-AMCA was detected under different conditions. First, the uptake of D-Ala-Lys-AMCA at different times $(0,15,30,60,120$ and $180 \mathrm{~min})$ but at the same $\mathrm{pH}$ value (6.0) and concentration $(25 \mu \mathrm{mol} / \mathrm{l})$ was detected. Second, D-Ala-Lys-AMCA uptake at different pH levels (5.4, $6,7.4$ and 8.4) but at the same time ( $2 \mathrm{~h}$ ) and concentrations ( $25 \mu \mathrm{mol} / \mathrm{l})$ was detected. Third, the effects of different initial concentrations of D-Ala-Lys-AMCA $(25,50$ and $150 \mu \mathrm{mol} / \mathrm{l})$ were analyzed, and these tests were performed at a $\mathrm{pH}$ of 6.0 for $2 \mathrm{~h}$. Inhibition tests were conducted by pre-incubating the cells with competitive compounds (Gly-Sar, Gly-Gln, Gly-Gly-Gly) for $30 \mathrm{~min}$ and then with D-Ala-Lys-AMCA $(25 \mu \mathrm{mol} / \mathrm{l})$ for $1 \mathrm{~h}$ at $37^{\circ} \mathrm{C}$ prior to the detection of fluorescence. Subsequent to removing the buffer and rapidly washing 3 times with ice-cold PBS, the cellular uptake of D-Ala-Lys-AMCA was examined with a BioTek Synergy 2 Multi-Mode reader (BioTek Instruments, Inc., Winooski, VT, USA) with excitation at $350 \mathrm{~nm}$, and emission at $460 \mathrm{~nm}$. All analyses were performed in triplicate.

Immunohistochemistry and immunofluorescence staining. A total of 82 human hepatocarcinoma tissue chips were purchased from Xi'an Alenabio Technology Co., Ltd. (Xi'an, China), which included 50 cases of hepatocarcinoma tissues (pathological grade 1,4 cases; grade 2, 20 cases; and grade 3, 26 cases), 13 cases of adjacent cancer tissues and 19 cases of normal liver tissues. Pathological grades 1, 2 and 3 were equivalent to well-, moderately- and poorly-differentiated, respectively (17). The expression of PEPT1 in these tissues was detected by immunohistochemistry. For immunohistochemical staining, formalin-fixed tissue samples were prepared as paraffin-embedded sections (thickness, $3 \mu \mathrm{m}$ ), and immunostaining of the sections was performed using the avidin-biotin-complex method: Primary antibody directed against PEPT1 (1:100 dilution) was diluted in PBS with $0.1 \%$ Tween and incubated with the sections overnight at $4^{\circ} \mathrm{C}$. The sections were then incubated with biotinylated secondary antibodies (dilution, 1:100; catalog no. ZB-2010; Beijing ZSGB-BIO Co. Ltd.) for $1 \mathrm{~h}$ at $37^{\circ} \mathrm{C}$, followed by the avidin-biotin complex for an additional $1 \mathrm{~h}$ at $37^{\circ} \mathrm{C}$. Protein expression was detected via coloration with 3,3'-diaminobenzidine in buffer, and the sections were counterstained with hematoxylin $(2 \mathrm{mg} / \mathrm{ml})$ at room temperature for $5 \mathrm{~min}$. Using a microcamera computational image analysis system (Cellsens standard; version 1.6; Olympus Corporation, Tokyo, Japan), a nucleus or cytoplasm containing brown-colored particles was considered positive. A total of five high-power fields were randomly selected for each group, and a total of 250 cells were counted. Sections with no labeling, or with $<5 \%$ labeled cells, were scored as 0 . Sections with $5-30 \%$ positive cells were scored as $1,31-70 \%$ positive cells as 2 , and $\geq 71 \%$ positive cells as 3 . Staining intensity was scored similarly, with 0 for negative staining, 1 for weakly positive, 2 for moderately positive, and 3 for strongly positive. Scores for the percentage of positive tumor cells and staining intensity were used to generate an immunoreactive score for each specimen. The quantity and intensity scores were calculated such that a final score of 0-1 indicated negative expression (-), 2-3 indicated weak expression $(+), 4-5$ indicated moderate expression $(++)$, and 6 indicated strong expression $(+++)(11)$.

Statistical analysis. Significance of Kaplan-Meier statistics was tested by calculating the log-rank. Data are expressed as the mean \pm standard deviation. SPSS software (version 16.0; SPSS, Inc., Chicago, IL, USA) was used for all calculations. $\mathrm{P}<0.05$ was considered to indicate a statistically significant difference.

\section{Results}

Immunofluorescence. Cell nuclei were labeled with blue fluorescence and red fluorescence indicated the presence of PEPT1 using the image system Cellsens standard (version 1.6; Olympus Corporation). All types of liver cancer cells emitted red fluorescence, as determined using microscopy (Fig. 1).

Western blotting. The results demonstrated that PEPT1 was expressed in the five liver cancer cell types studied (Bel-7402, SMMC7721, Hep3B, HepG2 and SK-HEP-1) to different degrees with SK-HEP-1 cells expressing the highest levels of PEPT1 (Fig. 1).

$R T-q P C R$. The results revealed that increased PEPT1 mRNA expression was present in the majority of liver cancer cells compared with Caco 2 cell lines. Furthermore, a markedly increased expression of the PEPT1 protein was observed in HepG2 cells compared with the other cell types, as determined by RT-qPCR (Fig. 1).

\section{Verification of D-Ala-Lys-AMCA}

Fluorescence of D-Ala-Lys-AMCA. D-Ala-Lys-AMCA is a well-known PEPT1 substrate that emits blue fluorescence. The results of the present study validated that D-Ala-Lys-AMCA may be transported into liver cancer and Caco- 2 cells, on the basis of the emission of blue fluorescence (Fig. 2).

Uptake of D-Ala-Lys-AMCA. The uptake of Ala-Lys-AMCA was time-dependent and also concentration-dependent, but not $\mathrm{pH}$-dependent. The maximum uptake occurred at a $\mathrm{pH}$ value of 7.4. Additionally, uptake was significantly decreased by the presence of Gly-Sar, Gly-Gln or Gly-Gly-Gly inhibitors (Fig. 3).

Immunohistochemistry. The results suggested that each group exhibited PEPT1 expression to different degrees (Fig. 4). Specifically, the expression of PEPT1 in hepatocarcinoma tissue was significantly higher compared with the expression observed in adjacent and normal liver tissue samples $(\mathrm{P}=0.0193$ and $\mathrm{P}=0.0057$, respectively, Table I). Significant differences in the expression of PEPT1 between three different pathological grades of liver cancer were observed, with tissues with a higher pathological grade demonstrating greater expression of PEPT1 ( $\mathrm{P}=0.0093$; Table II).

\section{Discussion}

Hepatocarcinoma is one of the most common malignant tumors worldwide (17). Each year, >700,000 incident patients are 


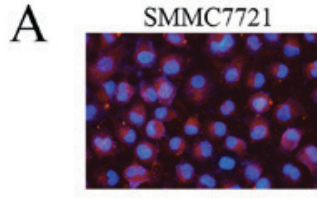

Hep3B

B
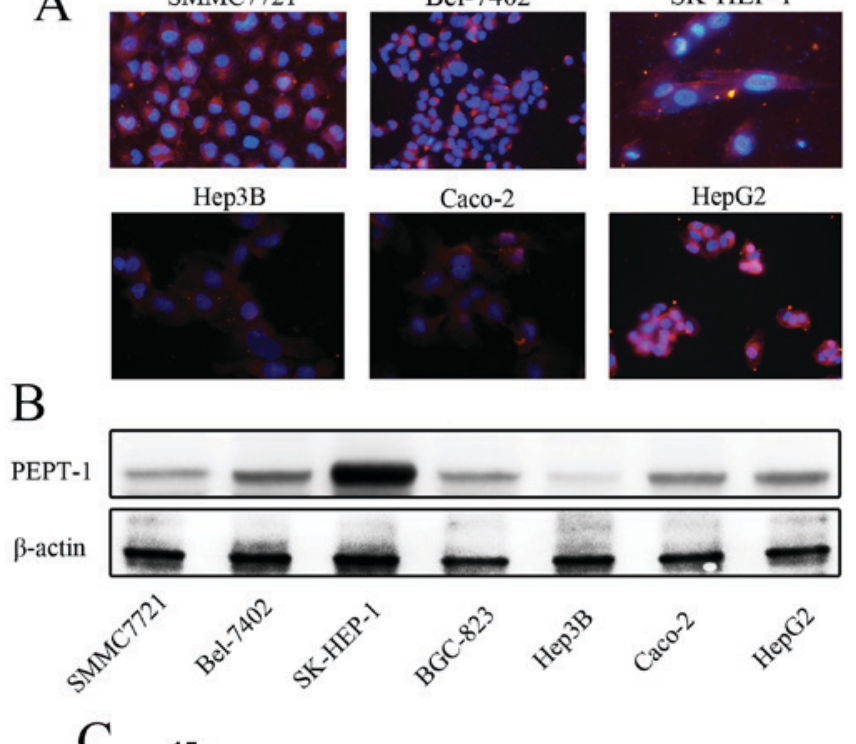

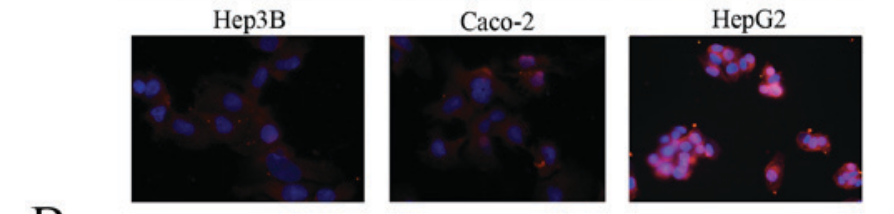

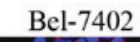

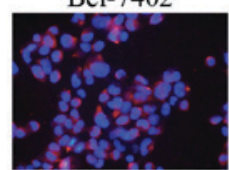

Caco-2

SK-HEP-1

HepG2
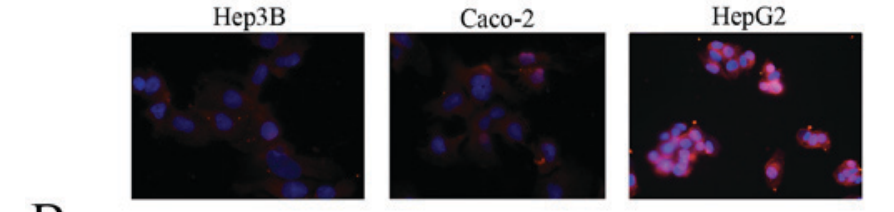

c

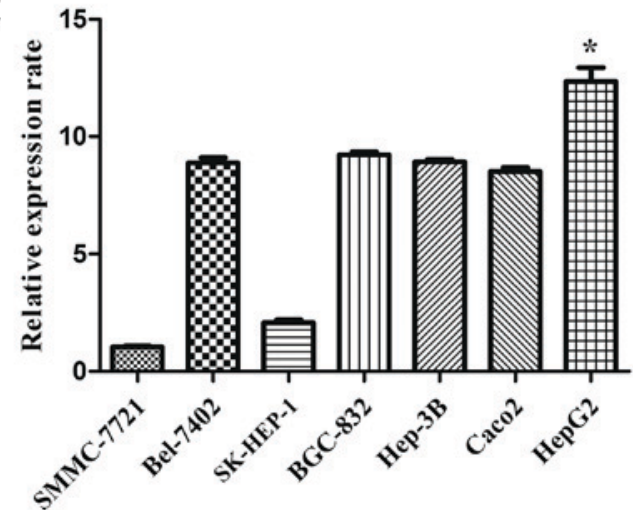

Figure 1. Expression of PEPT1 determined usingm (A) immunofluorescence (magnification, x200), (B) western blotting and (C) quantitative reverse transcription polymerase chain reaction in liver cancer cell lines (SMMC7721, Bel-7402, SK-HEP-1, Hep3B and HepG2). Caco-2 (human colon cancer cell line) and BGC-823 (human gastric cancer cell line) were used as positive controls. $\beta$-actin was used as an internal control for equal loading. PEPT1, proton-coupled oligopeptide transporter $1 .{ }^{*} \mathrm{P}<0.05$.

diagnosed, and 250,000 people succumb to liver cancer (18). In China, the hepatocarcinoma incidence is expected to markedly increase over the prospective decades, due to the increasing incidence of viral hepatitis infection, which is one of the most important pathogenic factors for the development of hepatocarcinoma (19). The preferred treatment for hepatocarcinoma is liver resection, but this treatment is restricted to patients with the very early stages of hepatocarcinoma (5). Due to the difficulty associated with achieving early diagnosis, the rapid progression of the disease and the fact that the majority of patients exhibit liver cirrhosis, few patients are able to undergo the operation, resulting in the majority of patients having a poor prognosis. Thus, non-operative therapy is an important treatment strategy for advanced hepatocarcinoma (19). At present, chemotherapy drugs commonly used in the clinical treatment of primary hepatocarcinoma include 5-fluorouracil, mitomycin, doxorubicin and epirubicin, among others (20). However, as chemotherapy is not specific to tumor cells, it may also affect normal cells and result in serious adverse effects. Transcatheter arterial chemoembolization and other local treatments may transport drugs directly to lesions, but
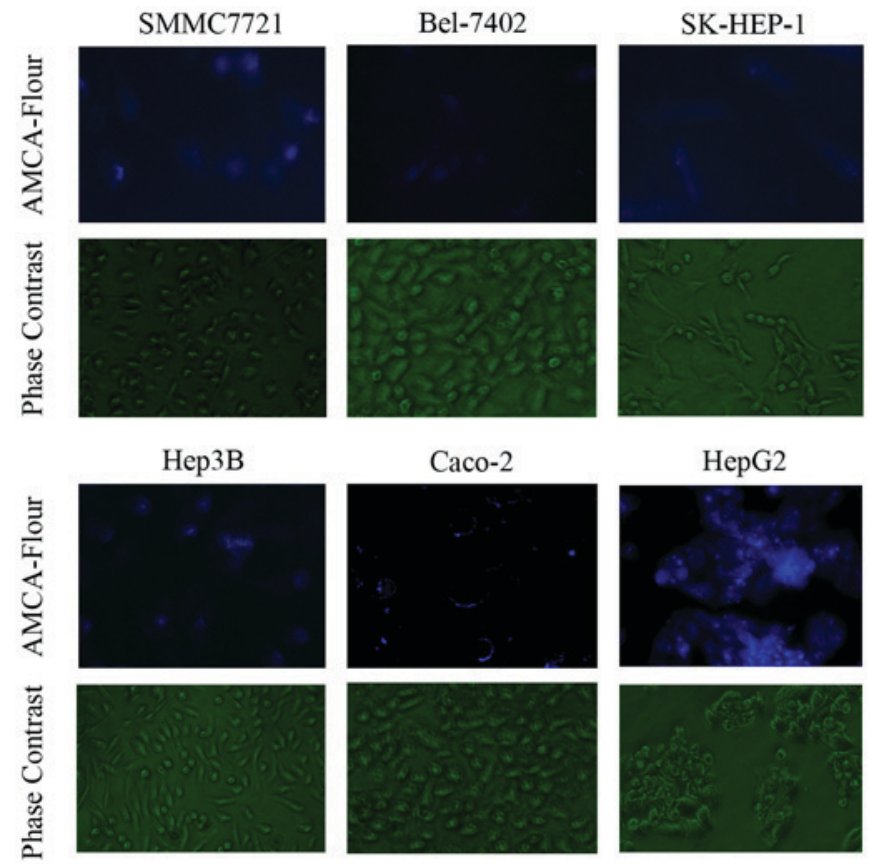

Figure 2. Visualization of proton-coupled oligopeptide transporter 1 in liver cancer cell lines based on the fluorescence of D-Ala-Lys-AMCA. Flour, fluorescent.

the effects of the dissemination of satellite foci and portal vein tumor thrombi are limited, and these therapies rarely control metastases that are distant from the lesions (21). Sorafenib is one targeted agent that serves an essential role in the treatment of advanced hepatocarcinoma, but high concentrations are required (22). Other novel targeted agents remain in the trial stage and require additional investigation (23). Therefore, the currently available therapies offer limited benefits to patients. As a result, there is a need for the development of novel and improved therapeutic strategies.

A targeted drug delivery system that may be used as an effective specific treatment may have good application prospects. In particular, targeted drug delivery is a system that uses a drug carrier that transports chemotherapy drugs to the specific location of a tumor, and may achieve directional and focal inhibition of the tumor cells, thus causing less injury to normal cells. This system has numerous advantages. For example, the drug maybe specifically transported to the target area, reach the maximum drug concentration in the target area, and react directly at the lesion site. Thus, this may promote the highest treatment effects with minimal toxic effects on normal cells, resulting in increased overall efficacy, safety and patient compliance with chemotherapy (24). The identification of a safe and effective targeted drug carrier is a focus of ongoing study.

Previous studies have suggested that peptide-targeted gold nanoparticles may serve as drug carriers for the treatment of brain cancer (25) and that biodegradable nanoparticles may deliver docetaxel to airway cancer cells in a targeted manner (26). In addition, a study of drug carriers for prostate cancer revealed that a peptide-drug conjugate exhibited markedly higher uptake by prostate cancer cells in comparison with the parent drug (27). Thus, the selection of an effective targeted carrier based on its specific oligopeptide transport activity is essential (28). If PEPT1 is specifically expressed in 
A $\quad$ B

B C

C
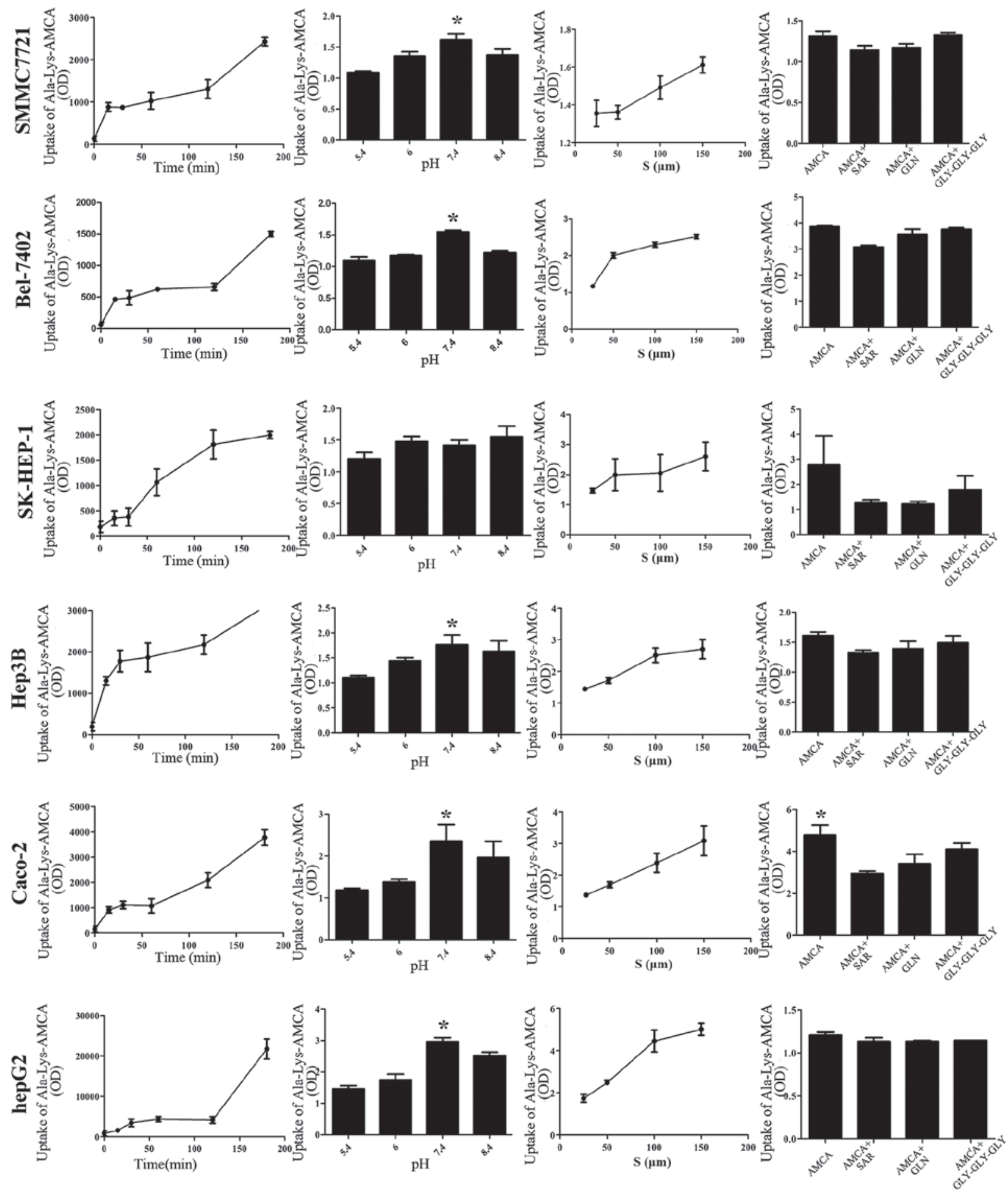

Figure 3. Uptake of Ala-Lys-AMCA under different conditions in five liver cancer cell types. (A) The uptake of D-Ala-Lys-AMCA at different times $(0,15,30,60,120$ and $180 \mathrm{~min})$, at $\mathrm{pH} 6.0$ and a concentration of $25 \mu \mathrm{mol} / 1$. (B) The uptake of D-Ala-Lys-AMCA at different pH values (5.4, 6.0, 7.4, 8.4) for $120 \mathrm{~min}$ at a concentration of $25 \mu \mathrm{mol} / 1$. (C) The uptake of D-Ala-Lys-AMCA at different concentrations ( 25,50 and $150 \mu \mathrm{mol} / \mathrm{l})$, at $\mathrm{pH} 6.0 \mathrm{for} 120 \mathrm{~min}$. (D) Inhibition tests with different competitive compounds (Gly-Sar, Gly-Gln, Gly-Gly-Gly). OD, optical density; S, substrates. P<0.05.

liver cancer cells and tissues, it may be a promising carrier for the delivery of chemotherapy drugs to a targeted region, with increased efficacy and decreased adverse effects on healthy tissues.

Peptides and peptide analogs may enter the cells of the body via peptide transporters in the membrane. The most widely studied peptide transporters are PEPT1 and PEPT2, which are members of the POT family (29). In mammals, the POT family comprises the following 4members, which are encoded by Solute carrier family 15 (SLC15) genes: PEPT1 (SLC15A1), PEPT2 (SLC15A2), peptide/histidine transporter 1 (PHT1; SLC15A4), and PHT2 (SLC15A3) (30). The functional 

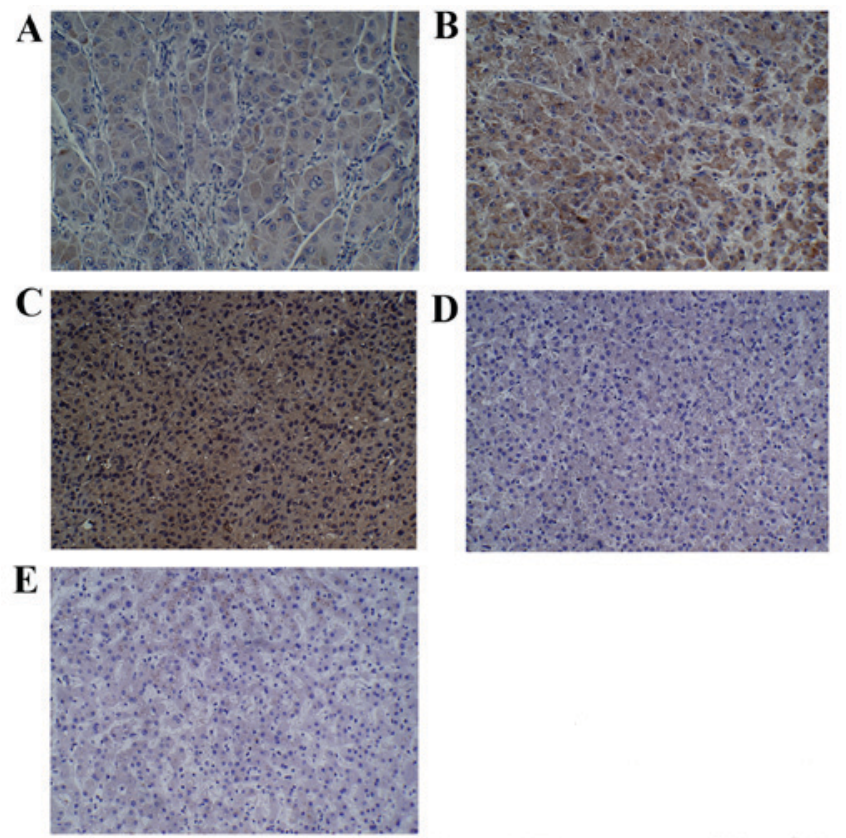

Figure 4. Expression of proton-coupled oligopeptide transporter 1 based on immunohistochemistry in different liver tissues (magnification, $\mathrm{x} 200$ ). (A) Pathological grade 1 liver cancer tissue. (B) Pathological grade 2 liver cancer tissue. (C) Pathological grade 3 liver cancer tissue. (D) Normal liver tissue. (E) Tissue adjacent to cancer.

expression of PEPT1 and PEPT2 has been identified: These peptide transporters are primarily expressed in the digestive tract and kidney, respectively $(31,32)$. The former demonstrates low affinity and high capacity, such that it may absorb a wide range of di/tripeptides $(33,34)$.

PEPT1, which is phylogenetically conserved, serves as an integral membrane protein in the cellular uptake of di/tripeptides and certain pharmacologically active drugs (35), and mediates the uptake of peptides and peptide-like molecules using the inwardly directed $\mathrm{H}^{+}$gradient across the membrane (36). PEPT1 is specifically a type of active transport protein with low affinity and high transport capacity that is almost exclusively expressed in humans, and several other mammalian species, including rats and mice $(37,38)$. This protein was first identified in the small intestine of a rabbit during cloning $(39,40)$. The PEPT1 molecule recognizes a wide range of oligopeptides and other compounds with similar structures, and its range of drug substrates is extensive, including $\beta$-lactam antibiotic drugs, angiotensin-converting enzyme inhibitors, antitumor and antiviral agents, thrombin inhibitors, dopamine receptor antagonists, and renin inhibitors $(41,42)$. The cellular uptake of these types of small peptides is an important physiological process mediated by proton-coupled peptide transporters (43). PEPT1 in particular may be involved in the transport of endogenous molecules and affects drug absorption, distribution, metabolism, and excretion, ultimately affecting the efficacy and toxicity of drugs. A loss of PEPT1 activity may therefore lead to a decrease in the intestinal absorption of di/tripeptides, peptidomimetics and peptide-like drugs (44). As PEPT1 is a proton-coupled carrier, it has a close association with the proton concentration. To summarize, oligopeptide transporters maybe regarded as putative therapeutic targets in cancer cells (45).
Table I. Expression of PEPT1 in different liver tissues.

\begin{tabular}{lcccccc}
\hline \multirow{2}{*}{$\begin{array}{l}\text { Tissue } \\
\text { type }\end{array}$} & & \multicolumn{3}{c}{ PEPT1 expression } & \\
\cline { 3 - 5 } & $\mathrm{n}$ & - & + & ++ & +++ & $\begin{array}{l}\text { Positive } \\
\text { rate, \% }\end{array}$ \\
\hline Hepatocarcinoma & 50 & 7 & 6 & 12 & 25 & 86.00 \\
Adjacent cancer & 13 & 6 & 4 & 2 & 1 & $53.85^{\mathrm{a}}$ \\
Normal liver & 19 & 9 & 5 & 4 & 1 & $52.63^{\mathrm{b}}$ \\
\hline
\end{tabular}

SPSS version 16.0 was used for all calculations. ${ }^{\mathrm{a}} \mathrm{P}=0.0193$ hepatocarcinoma vs. adjacent cancer tissue; ${ }^{\mathrm{b}} \mathrm{P}=0.0057$ hepatocarcinoma vs. normal liver tissue; -, negative expression; +, weak expression; ++, moderate expression; +++, strong expression; PEPT1, proton-coupled oligopeptide transporter 1

Table II. Expression of PEPT1 in different pathological hepatocarcinoma grades.

\begin{tabular}{lrrrrrr}
\hline & & \multicolumn{3}{c}{ PEPT1 expression } & \\
\cline { 3 - 5 } $\begin{array}{l}\text { Pathological } \\
\text { grade }\end{array}$ & $\mathrm{n}$ & - & + & ++ & +++ & $\begin{array}{c}\text { Positive } \\
\text { rate, \% }\end{array}$ \\
\hline 1 & 4 & 3 & 1 & 0 & 0 & 25.00 \\
2 & 20 & 2 & 2 & 6 & 10 & 90.00 \\
3 & 26 & 2 & 3 & 6 & 15 & $92.31^{\mathrm{a}}$ \\
\hline
\end{tabular}

SPSS version 16.0 was used for calculation. ${ }^{\mathrm{a}} \mathrm{P}=0.0093$ grade 1 vs. grade 3 ; -, negative expression; +, weak expression; ++, moderate expression; +++, strong expression; PEPT1, proton-coupled oligopeptide transporter 1 .

The results of the present study demonstrated that PEPT1 has relatively limited expression in normal tissues, but is highly expressed in various types of tumor cells $(46,47)$. PEPT1 was already known to exhibit high expression in the pancreatic cancer cell lines AsPC-1 and Capan-2, and low expression in adjacent tissues $(47,48)$. Nakanishi et al (15) first revealed the expression of PEPT1 in the human fibro sarcoma HT1080 cell line. In addition, the expression of PEPT1 in the gastric cancer MKN45 cell line was previously suggested (49), and an additional study identified high expression of PEPT1 in prostate cancer cells (13). However, at present, little is known about the expression of PEPT1 in primary hepatocarcinoma or its significance for targeted drug delivery.

Caco-2 is a human colon cancer cell line that was used as a positive control in the present study as it is generally considered o exhibit high expression of PEPT1 $(50,51)$. In the immunofluorescence analysis, it was observed that the PEPT1 protein (red fluorescence) was localized to the plasma membrane of the liver cancer cells Bel-7402, SMMC7721, Hep3B, HepG2 and SK-HEP-1, similar to what was observed for Caco-2 cells. These results directly demonstrated the expression of PEPT1 in liver cancer.

A previous study identified the expression of PEPT1 in gastric cancer cells (12). In the present study, the expression of PEPT1 in the gastric cancer cell line BGC-823 was examined, and the results were consistent with a previous study (12). In 
the case of BGC-823 and Caco-2 cells, which were used as a positive control, universal expression of PEPT1 was observed, similar to data previously demonstrated for PEPT1 in other cancer cells (11-15). Although PEPT1 demonstrated different functional activities in the liver cell lines, the expression of PEPT1 in SK-HEP-1 cells was highest, as determined by western blotting. In contrast, the highest expression detected by RT-qPCR was observed in HepG2 cells. The potential for experimental error was eliminated by repeating experiments three times. The reasons underlying this discrepancy may be associated with differences in cellular status, the presence of protein isoforms and the regulation of protein transcription or translation.

To study the functional activity of PEPT1 in liver cancer cells and to determine the role of PEPT1 in the uptake of PEPT1 substrates, the specific fluorescence substrate Ala-Lys-AMCA, a well-known PEPT1 substrate, was studied in the absence and presence of PEPT1 (30). The fluorescence analysis of D-Ala-Lys-AMCA confirmed that D-Ala-Lys-AMCA may be transported into liver cancer cells and Caco-2 cells, which indirectly demonstrated the expression of PEPT1 in hepatocarcinoma cells. A previous study investigated the function of PEPT1 in the mouse intestine through electrophysiological methods (52). In the present study, the absorption of substrates at different times, $\mathrm{pH}$ values and concentrations were determined. It was also identified that the uptake of Ala-Lys-AMCA was time- and concentration-dependent. All of these data confirm that the transport of PEPT1 may be affected by time, $\mathrm{pH}$ and substrate inhibitors, as observed in previous studies $(53,54)$. Gly-Sar is a small peptide that also serves as a substrate for PEPT1, which specifically recognizes and transports it (47). A study conducted by Berthelsen et al (55) demonstrated that basolateral Gly-Sar transport in the intestinal cell line Caco-2 is specifically proton-coupled via PEPT1. The dipeptide Gly-Gln is also known as a high-affinity substrate for PEPT1, which transports it into the cell in an inward direction (13). In the present study, Gly-Sar, Gly-Gln and Gly-Gly-Gly were all used as competitive substrates in a competition inhibition test $(56,57)$, which demonstrated that the uptake of D-Ala-Lys-AMCA was significantly decreased by all three inhibitors. Thus, this suggests that the liver cancer cells examined expressed functionally active PEPT1 in the plasma membrane, and that PEPT1 serves an important role in the transport of the substrate Ala-Lys-AMCA.

A tissue microarray analysis was performed in the present study to provide a preliminary investigation of the expression of PEPT1 in normal liver tissues, liver cancer tissues with different pathological grades and tissues adjacent to liver cancer. The analysis demonstrated that the expression of PEPT1 in cancer tissues was higher compared with that in normal tissues $(\mathrm{P}<0.05)$, whereas a low expression of PEPT1 was observed in adjacent tissues. In addition, the expression levels were associated with the pathological grade of the liver cancer tissues. In summary, it was demonstrated that PEPT1 is expressed in liver cancer tissues, and that PEPT1 overexpression is associated with more aggressive tumor malignancy and a poor prognosis. Therefore, PEPT1 may serve as an indicator of the nature of liver cancer (benign or malignant) and of the differentiation degree of liver cancer cells, making it an attractive target for cancer therapy.
In the present study, initial exploration of the specific overexpression of PEPT1 in primary hepatocarcinoma cells and liver cancer tissues was performed using various approaches, and different conditions. The correlation between tumor tissues and PEPT1 indicates that PEPT1 represents a promising molecular target for targeted drug delivery.

The selection of a drug carrier must meet the following criteria: First, the carrier should be able to transport the drug into the body and to avoid attack by the immune systems of the body. Secondly, the carrier should deliver the drug specifically to a particular location and cell type, and should guarantee drug release (58). Furthermore, the targeted drug should exhibit the highest bioavailability possible, and reach its appropriate site of action through efficient transport by drug carriers (59).

Current tumor therapy primarily relies on highly toxic chemical drugs, which lead to numerous serious side effects. For example, paclitaxel and doxorubicin may induce neurotoxicity, cardiac toxicity and bone marrow suppression during treatment of a tumor. An effective approach to reduce the side effects of chemotherapy would be the selective delivery of anticancer drugs to tumor tissues (60).

As its substrate-binding site may accommodate a wide range of molecules of different sizes, hydrophobicities and charges, PEPT1is regarded as an excellent target for the delivery of pharmacologically-active compounds (61).

Due to its high expression in hepatocarcinoma, PEPT1 may serve as a good transporter-mediated drug delivery target, to improve the treatment of primary hepatocarcinoma. Generally, the design of a drug carrier that may specifically deliver drugs to a tumor site is of importance. Therefore, the present study hypothesized that a chemotherapy drug may be modified to obtain a structure similar to that of di/tripeptides, such that it may be identified and transported into hepatocarcinoma cells by PEPT1 in a targeted manner. This approach may constitute a potential therapeutic strategy for cancer treatment and may lead to the development of a chemotherapy drug with increased efficacy, and reduced toxic effects on the heart and bone marrow. Therefore, PEPT1 represents a novel direction for future study, and as the next stage, cell and animal experiments should be performed to additionally examine the aforementioned theory. In addition, all of the hypotheses discussed in the present study require additional verification.

\section{Acknowledgements}

The present study was supported by the National Natural Science Foundation of China (grant no. 81302081).

\section{References}

1. Zong G, Xu Z, Zhang S, Shen Y, Qiu H, Zhu G, He S, Tao T and Chen X: CD109 mediates cell survival in hepatocellular carcinoma cells. Dig Dis Sci 61: 2303-2314, 2016.

2. Mansourian PG, Yoneda M, Krishna Rao M, Martinez FJ, Thomas E and Schiff ER: Effects of statins on the risk of hepatocellular carcinoma. Gastroenterol Hepatol (N Y) 10: 417-426, 2014.

3. Kew MC: Hepatocellular carcinoma in developing countries: Prevention, diagnosis and treatment. World J Hepatol 4: 99-104, 2012. 
4. Zhang S, Yue M, Shu R, Cheng H and Hu P: Recent advances in the management of hepatocellular carcinoma. J BUON 21: 307-311, 2016.

5. Harlan LC, Parsons HM, Wiggins CL, Stevens JL and Patt YZ: Treatment of hepatocellular carcinoma in the community: Disparities in standard therapy. Liver Cancer 4: 70-83, 2015.

6. Yuen MF, Ahn SH, Chen DS, Chen PJ, Dusheiko GM, Hou JL, Maddrey WC, Mizokami M, Seto WK, Zoulim F and Lai CL: Chronic Hepatitis B virus infection: Disease revisit and management recommendations. J Clin Gastroenterol 50: 286-294, 2016.

7. Parker JL, Mindell JA and Newstead S: Thermodynamic evidence for a dual transport mechanism in a POT peptide transporter. Elife 3, 2014

8. Jensen JM, Simonsen FC, Mastali A, Hald H, Lillebro I, Diness F, Olsen L and Mirza O: Biophysical characterization of the proton-coupled oligopeptide transporter YjdL. Peptides 38 89-93, 2012.

9. Gong Y, Wu X, Wang T, Zhao J, Liu X, Yao Z, Zhang Q and Jian X: Targeting PEPT1: A novel strategy to improve the antitumor efficacy of Doxorubicin in human hepatocellular carcinoma therapy. Oncotarget 15: 40454-40468, 2017.

10. Kumar KK, Karnati S, Reddy MB and Chandramouli R: Caco-2 cell lines in drug discovery-an updated perspective. J Basic Clin Pharm 1: 63-69, 2010.

11. Gonzalez DE, Covitz KM, Sadée W and Mrsny RJ: An oligopeptide transporter is expressed at high levels in the pancreatic carcinoma cell lines AsPc-1 and Capan-2. Cancer Res 58: 519-525, 1998.

12. Namikawa T, Yatabe T, Inoue $K$, Shuin T and Hanazaki K: Clinical applications of 5-aminolevulinic acid-mediated fluorescence for gastric cancer. World J Gastroenterol 21: 8769-8775, 2015.

13. Tai W, Chen $\mathrm{Z}$ and Cheng K: Expression profile and functional activity of peptide transporters in prostate cancer cells. Mol Pharm 10: 477-487, 2013.

14. Sun D, Tan F, Fang D, Wang Y, Zeng S and Jiang H: Expression of proton-coupled oligopeptide transporter (POTs) in prostate of mice and patients with benign prostatic hyperplasia (BPH) and prostate cancer (PCa). Prostate 73: 287-295, 2013.

15. Nakanishi T,Tamai I,Sai Y,Sasaki TandTsuji A:Carrier-mediated transport of oligopeptides in the human fibrosarcoma cell line HT1080. Cancer Res 57: 4118-4122, 1997.

16. Livak KJ and Schmittgen TD: Analysis of relative gene expression data using real time quantitative PCR and the 2(-Delta Delta C(T)) method. Methods 25: 402-408, 2001.

17. Gong XL and Qin SK: Progress in systemic therapy of advanced hepatocellular carcinoma. World J Gastroenterol 22: 6582-6594, 2016.

18. Slotta JE, Kollmar O, Ellenrieder V, Ghadimi BM and Homayounfar K: Hepatocellular carcinoma: Surgeon's view on latest findings and future perspectives. World J Hepatol 7: $1168-1183,2015$

19. Wang CH, Wey KC, Mo LR, Chang KK, Lin RC and Kuo JJ: Current trends and recent advances in diagnosis, therapy and prevention of hepatocellular carcinoma. Asian Pac J Cancer Prev 16: 3595-3604, 2015

20. Sahara S, Kawai N, Sato M, Tanaka T, Ikoma A, Nakata K, Sanda H, Minamiguchi H, Nakai M, Shirai S and Sonomura T: Prospective evaluation of transcatheter arterial chemoembolization (TACE) with multiple anti-cancer drugs (epirubicin cisplatin, mitomycin c,5-fluorouracil) compared with TACE with epirubicin for treatment of hepatocellular carcinoma. Cardiovasc Intervent Radiol 35: 1363-1371, 2012.

21. Paul SB and Sharma H: Role of transcatheter intra-arterial therapies for hepatocellular carcinoma. J Clin Exp Hepatol 4 (Suppl 3): S112-S121, 2014

22. Keating GM: Sorafenib: A Review in hepatocellular carcinoma Target Oncol 12: 243-253, 2017.

23. Deng GL, Zeng S and Shen H: Chemotherapy and target therapy for hepatocellular carcinoma: New advances and challenges. World J Hepatol 7: 787-798, 2015.

24. Zheng C, Ma C, Bai E, Yang $\mathrm{K}$ and Xu R: Transferrin and cell-penetrating peptide dual-functioned liposome for targeted drug delivery to glioma. Int J Clin Exp Med 8: 1658-1668, 2015 .

25. Meyers JD, Cheng Y, Broome AM, Agnes RS, Schluchter MD, Margevicius S, Wang X, Kenney ME, Burda C and Basilion JP: Peptide-targeted gold nanoparticles for photodynamic therapy of brain cancer. Part Part Syst Charact 32: 448-457, 2015.
26. Maiolino S, Russo A, Pagliara V, Conte C, Ungaro F, Russo G and Quaglia F: Biodegradable nanoparticles sequentially decorated with Polyethyleneimine and Hyaluronan for the targeted delivery of docetaxel to airway cancer cells. J Nanobiotechnology 13: 29, 2015.

27. Tai W, Shukla RS, Qin B, Li B and Cheng K: Development of a peptide-drug conjugate for prostate cancer therapy. Mol Pharm 8: 901-912, 2011.

28. Nakanishi T, Tamai I, Takaki A and Tsuji A: Cancer cell-targeted drug delivery utilizing oligopeptide transport activity. Int J Cancer 88: 274-280, 2000.

29. Nielsen CU and Brodin B: Di/tri-peptide transporters as drug delivery targets: Regulation of transport under physiological and patho-physiological conditions. Curr Drug Targets 4: 373-388, 2003.

30. Sun D, Wang Y, Tan F, Fang D, Hu Y, Smith DE and Jiang H: Functional and molecular expression of the proton-coupled oligopeptide transporters in spleen and macrophages from mouse and human. Mol Pharm 10: 1409-1416, 2013.

31. Daniel H and Kottra G: The proton oligopeptide cotransporter family SLC15 in physiology and pharmacology. Pflugers Arch 447: 610-618, 2004.

32. Ganapathy ME, Huang W, Wang H, Ganapathy V and Leibach FH: Valacyclovir: A substrate for the intestinal and renal peptide transporters PEPT1 and PEPT2. Biochem Biophys Res Commun 246: 470-475, 1998

33. Sugawara M, Huang W, Fei YJ, Leibach FH, Ganapathy V and Ganapathy ME: Transport of valganciclovir, a ganciclovir prodrug, via peptide transporters PEPT1 and PEPT2. J Pharm Sci 89: 781-789, 2000

34. Rubio-Aliaga I and Daniel H: Peptide transporters and their roles in physiological processes and drug disposition. Xenobiotica 38 : 1022-1042, 2008

35. Hu Y, Xie Y, Keep RF and Smith DE: Divergent developmental expression and function of the proton-coupled oligopeptide transporters PepT2 and PhT1 in regional brain slices of mouse and rat. J Neurochem 129: 955-965, 2014.

36. Doki S, Kato HE, Solcan N, Iwaki M, Koyama M, Hattori M, Iwase N, Tsukazaki T, Sugita Y, Kandori H, et al: Structural basis for dynamic mechanism of proton-coupled symport by the peptide transporter POT. Proc Natl Acad Sci USA 110: $11343-11348,2013$.

37. Hu Y, Xie Y, Wang Y, Chen X and Smith DE: Development and characterization of a novel mouse line humanized for the intestinal peptide transporter PEPT1. Mol Pharm 11: 3737-3746, 2014.

38. Yang B, Hu Y and Smith DE: Impact of peptide transporter 1 on the intestinal absorption and pharmacokinetics of valacyclovir after oral dose escalation in wild-type and PepT1 knockout mice. Drug Metab Dispos 41: 1867-1874, 2013.

39. Boll M, Markovich D, Weber WM, Korte H, Daniel H and Murer H: Expression cloning of a cDNA from rabbit small intestine related to proton-coupled transport of peptides, beta-lactam antibiotics and ACE-inhibitors. Pflugers Arch 429: 146-149, 1994.

40. Hsu CP, Hilfinger JM, Walter E, Merkle HP, Roessler BJ and Amidon GL: Overexpression of human intestinal oligopeptide transporter in mammalian cells via adenoviral transduction. Pharm Res 15: 1376-1381, 1998.

41. Terada T and Inui K: Peptide transporters: Structure, function, regulation and application for drug delivery. Curr Drug Metab 5: 85-94, 2004.

42. Luckner $\mathrm{P}$ and Brandsch $\mathrm{M}$ : Interaction of 31 beta-lactam antibiotics with the $\mathrm{H}+$ /peptide symporter PEPT2: Analysis of affinity constants and comparison with PEPT1. Eur J Pharm Biopharm 59: 17-24, 2005.

43. Newstead S: Molecular insights into proton coupled peptide transport in the PTR family of oligopeptide transporters. Biochim Biophys Acta 1850: 488-499, 2015.

44. Jappar D, Wu SP, Hu Y and Smith DE: Significance and regional dependency of peptide transporter (PEPT) 1 in the intestinal permeability of glycylsarcosine: In situ single-pass perfusion studies in wild-type and Pept1 knockout mice. Drug Metab Dispos 38: 1740-1746, 2010.

45. Mrsny RJ: Oligopeptide transporters as putative therapeutic targets for cancer cells. Pharm Res 15: 816-818, 1998.

46. Landowski CP, Vig BS, Song X and Amidon GL: Targeted delivery to PEPT1-overexpressing cells: Acidic, basic and secondary floxuridine amino acid ester prodrugs. Mol Cancer Ther 4: 659-467, 2005. 
47. Mitsuoka K, Kato Y, Miyoshi S, Murakami Y, Hiraiwa M, Kubo Y, Nishimura S and Tsuji A: Inhibition of oligopeptide transporter suppress growth of human pancreatic cancer cells. Eur J Pharm Sci 40: 202-208, 2010.

48. Gonzalez DE, Covitz KM, Sadée W and Mrsny RJ: An oligopeptide transporter is expressed at high levels in the pancreatic carcinoma cell lines AsPc-1 and Capan-2. Cancer Res 58: 519-525, 1998.

49. Inoue M, Terada T, Okuda M and Inui K: Regulation of human peptide transporter 1 (PEPT1) in gastric cancer cells by anticancer drugs. Cancer Lett 230: 72-80, 2005.

50. Buyse M, Berlioz F, Guilmeau S, Tsocas A, Voisin T, Péranzi G, Merlin D, Laburthe M, Lewin MJ, Rozé C and Bado A: PepT1-mediated epithelial transport of dipeptides and cephalexin is enhanced by luminal leptin in the small intestine. J Clin Invest 108: 1483-1494, 2001.

51. Han HK, Oh DM and Amidon GL: Cellular uptake mechanism of amino acid ester prodrugs in Caco-2/hPEPT1 cells overexpressing a human peptide transporter. Pharm Res 15: 1382-1386, 1998.

52. Chen M, Singh A, Xiao F, Dringenberg U, Wang J, Engelhardt R, Yeruva S, Rubio-Aliaga I, Nässl AM, Kottra G, et al: Gene ablation for PEPT1 in mice abolishes the effects of dipeptides on small intestinal fluid absorption, short-circuit current, and intracellular pH. Am J Physiol Gastrointest Liver Physiol 299: G265-G274, 2010.

53. Verri T, Kottra G, Romano A, Tiso N, Peric M, Maffia M, Boll M, Argenton F, Daniel H and Storelli C: Molecular and functional characterisation of the zebrafish (Danio rerio) PEPT1-type peptide transporter. FEBS Lett 549: 115-122, 2003.

54. Agu R, Cowley E, Shao D, Macdonald C, Kirkpatrick D, Renton K and Massoud E: Proton-coupled oligopeptide transporter (POT) family expression in human nasal epithelium and their drug transport potential. Mol Pharm 8: 664-672, 2011.
55. Berthelsen R, Nielsen CU and Brodin B: Basolateral glycylsarcosine (Gly-Sar) transport in Caco-2 cell monolayers is $\mathrm{pH}$ dependent. J Pharm Pharmacol 65: 970-979, 2013.

56. Dalmasso G, Nguyen HT, Charrier-Hisamuddin L, Yan Y, Laroui H, Demoulin B, Sitaraman SV and Merlin D: PepT1 mediates transport of the proinflammatory bacterial tripeptide L-Ala-\{gamma\}-D-Glu-meso-DAP in intestinal epithelial cells. Am J Physiol Gastrointest Liver Physiol 299: G687-G696, 2010.

57. Terada T, Sawada K, Ito T, Saito H, Hashimoto Y and Inui K: Functional expression of novel peptide transporter in renal basolateral membranes. Am J Physiol Renal Physiol 279: F851-F857, 2000.

58. Tan S, Wu T, Zhang D and Zhang Z: Cell or cell membrane-based drug delivery systems. Theranostics 5: 863-881, 2015.

59. Foltz M, Meyer A, Theis S, Demuth HU and Daniel H: A rapid in vitro screening for delivery of peptide-derived peptidase inhibitors as potential drug candidates via epithelial peptide transporters. J Pharmacol Exp Ther 310: 695-702, 2004.

60. Wang S, Placzek WJ, Stebbins JL, Mitra S, Noberini R, Koolpe M Zhang Z, Dahl R, Pasquale EB and Pellecchia M: Novel targeted system to deliver chemotherapeutic drugs to EphA2-expressing cancer cells. J Med Chem 55: 2427-2436, 2012.

61. Rubio-Aliaga I and Daniel H: Mammalian peptide transporters as targets for drug delivery. Trends Pharmacol Sci 23: 434-440, 2002. 